\title{
FORTHCOMING IN THE JOURNAL OF ECONOMETRICS Panel Estimators and the Identification of Firm-Specific Efficiency Levels in Parametric, Semiparametric and Nonparametric Settings*
}

\author{
Robin C. Sickles \\ Department of Economics \\ Rice University
}

July 6, 2004

\begin{abstract}
The paper analyzes a number of competing approaches to modeling efficiency in panel studies. The specifications considered include the fixed effects stochastic frontier, the random effects stochastic frontier, the Hausman-Taylor random effects stochastic frontier, and the random and fixed effects stochastic frontier with an $\mathrm{AR}(1)$ error. I have summarized the foundations and properties of estimators that have appeared elsewhere and have described the model assumptions under which each of the estimators have been developed. I discuss parametric and nonparametric treatments of time varying efficiency including the BatteseCoelli estimator and linear programming approaches to efficiency measurement. Monte Carlo simulation is used to compare the various estimators and to assess their relative performances under a variety of misspecified settings. A brief illustration of the estimators is conducted using U. S. banking data.
\end{abstract}

Keywords: Nonparametric methods, productivity, frontier production

JEL Classification Numbers: C14, D24

\footnotetext{
*The author would like to thank Jeffrey Dorfman, three anonymous referees, Daniel Rich and participants at the October 2002 Conference on Current Developments in Productivity and Efficiency Measurement, organized by Jeffrey Dorfman and Gary Koop, for their insightful comments as well as participants at the 2nd Helenic Workshop on Efficiency and Productivity Measurement, University of Patras, May/June 2003. Bill Greene provided needed criticisms on this draft. Thanks are also extended to Joon Park and Yoosoon Chang as well as participants in the econometrics workshop at the University of Korea. Wonho Song provided essential research assistance. The usual caveat applies.
} 


\section{Introduction}

Since the fundamental theoretical work by Debreu (1951), Farrell (1957), Shephard (1953, 1970) and Afriat (1972), researchers have established a method to measure the intrinsically unobservable phenomena of efficiency. Aigner et al. (1977), Battese and Cora (1977), and Meeusen and van den Broeck (1977) provided the econometric methods for the applications waiting to happen. The linear programming methodology, whose implementation was made transparent by Charnes et al. (1978), became available at about the same time. The U. S. and international emphasis on deregulation and the efficiencies accruing to increased international competition from lower trade barriers has provided a fertile research experiment for efficiency modelers and practitioners.

The efficiency score, as it is usually measured, is a residual. Parametric assumptions about the distribution of efficiency and its correlation structure often are made to sharpen the interpretation of the residual. Work by a number of scholars in the area has focused on such parametric models. We consider a number of these models below. However, that efficiency measurement should be highly leveraged by parametric assumptions is by no means a comforting resolution to this measurement problem. Productivity defined by the Solow residual is a reduced form concept, not one that can be given a structural interpretation without a more formal structural model. It is what it is, a residual, and the various stochastic frontier efficiency estimators that use a particular decomposition of cross-sectional and temporal variation in the Solow residual are what they are, various reduced form estimators of the residual. Among the more important distinguishing features of competing estimators of efficiency are their flexibility, robustness, and

precision. Given the ad hoc nature of most parametric specifications it may be instructive to consider a number of alternative estimators that possess the generic properties of fliexibility, robustness, and precision when conducting empirical efficiency analyses.

In this paper I have summarized the foundations and properties of estimators that have appeared elsewhere and have described the model assumptions under which each of the estimators has been developed. I analyze traditional and recently developed efficiency estimators. The recently developed estimators include the class of semiparametric efficient estimators that make minimal assumptions about the distribution of unobserved efficiencies. The estimators we study are developed in a panel data setting. Cross-sectional data models are not explored. The semiparametric efficient estimators share a number of generic properties with the estimators proposed by Schmidt and Sickles (1984) and Cornwell et al. (1990) and can be viewed as alternatives to the parametric models of Pitt and Lee (1982), Kumbhakar (1990), Battese and Coelli (1992), Coelli et al. (1998). The semiparametric efficient class of efficiency estimators provides an alternative to fully parametric stochastic frontiers and to nonparametric estimators of efficiency based on linear programming characterizations of technology. We impose structure on the class of semiparametric efficient estimators (SPE) by utilizing a linear in logs (or geometric mean in inputs) parameterization of technology. For this relatively simple technology structure we ask how well will the robust procedures identify and estimate the efficiency scores of particular firms at a point in time and over time under various forms of misspecification? If we have a difficult time providing convincing arguments that such possibilities are encouraging 
for a relatively simple characterization of the empirical production relationship, then extensions to more complicated technologies are problematic. ${ }^{1}$ Results from the Monte Carlo simulations indicate that it is indeed difficult to identify firm-specific and temporally varying efficiencies and that a strong institutional understanding of the industry under study is crucial to understanding which estimator should be utilized.

The plan of the paper is as follows. In Section 2 we discuss issues of specification and estimation in SPE specification of the panel stochastic frontier model and outline the differing assumptions that distinguish the set of existing and newly derived semiparametric efficient estimators for the panel frontier model and for the efficiency effect. Discussion of a set of alternatives to the models in the SPE class is provided in Section 3. A number of these methods have appeared in the literature and are widely used by practitioners. The competing estimators are analyzed via Monte Carlo simulations of an underlying inefficient technology in Section 4. An empirical illustration of efficiency using a subsample of U. S. Banking firms during the deregulatory period of the 1980s is presented in Section 5. Concluding remarks are provided in Section 6.

\section{The Semiparametric Model and Estimators of Technical Effi- ciency}

The basic model we analyze can be written as:

$$
Y_{i t}=X_{i t}^{\prime} \beta+\alpha_{i}+\varepsilon_{i t} \quad i=1, \ldots, N ; t=1, \ldots, T
$$

where $X_{i t} \in \mathbb{R}^{d}, \beta \in \mathbb{R}^{d}$. The $\varepsilon_{i t}$ are assumed to be random variables from an $N\left(0, \sigma^{2}\right)$. In Section 3 we consider cases in which the $\varepsilon_{i t}$ are iid and in which they are serially dependent. Let $Y_{i}=\left(Y_{i 1}, \ldots, Y_{i T}\right)^{\prime}, X_{i}=\left(X_{i 1}^{\prime}, \ldots, X_{i T}^{\prime}\right)^{\prime}$. The $\left(\alpha_{i}, X_{i}\right)$ 's are assumed to be iid random variables having unknown density $h(\cdot, \cdot)$ on $\mathbb{R}^{1+d T}$. The unknown density is specified in the derivations of the semiparametric efficient estimators using kernel smoothers. The support of the marginal density of $\alpha$ is assumed to be bounded above (or below), where, for example, the bound $B$ provides the upper level of the production frontier or the lower level of the cost frontier. We consider cases in which $\varepsilon$ 's and $(\alpha, X)$ 's are independent as well as cases in which certain dependency structures exist. This generic panel model can be reinterpreted as a stochastic panel production frontier model (Pitt and Lee, 1981; Schmidt and Sickles, 1984) if $Y_{i t}$ is the $t$-th observation on the logarithm of output of the $i$-th firm, $X_{i t}$ is a vector of the $t$-th observation of the logarithm of the $d$ inputs of the $i$-th firm and $\alpha_{i}$ is an unobservable random effect that captures firm-specific inefficiency. With panel data, realizations of $\alpha_{i}$ for a particular firm

\footnotetext{
${ }^{1}$ This paper does not consider the class of models and estimators for such models that address the identification of allocative and technical efficiency. These models are highly parametric and rely on strong distributional assumptions for identification. Models of this sort have been considered by, among others, Lau and Yotopoulos (1971), Toda (1976), Atkinson and Halvorsen (1980), Lovell and Sickles (1983), Sickles et al. (1986), Kumbhakar (1987), Good et al. (1991), Atkinson and Cornwell (1994), and Good et al. (1997). For an excellent review of this class of models as well as the methods we address herein see Greene (1997) and Lovell and Kumbhakar (2000).
} 
can be identified, thus overcoming the limitation of a single cross-section (or time series) from which one can only identify the expectation of $\alpha_{i}$ conditional on stochastic noise (Jondrow et al., 1982). Normality of the within disturbance term can be based on central limit arguments. Motivation for a particular parametric distribution of the inefficiency terms is less easily justified. Although the distribution of the inefficiency term is one-sided, the terms are intrinsically latent and unobservable components. Flexible one-sided distributions such as the gamma have been proposed by Greene (2003). There are continuing practical questions on the identifiability of the parameters of such a model (Ritter and Simar, 1997), however, and they have not yet been used widely in panel studies. The SPE estimators we discuss below are designed to provide robust point estimates for the parameters that describe the technology while assuring the smallest standard errors for slope parameter estimates. The efficiency measurements we pursue are residual based and thus have properties that are leveraged on the properties of the slope parameters which are used in their construction.

In order to understand how the semiparametric efficient estimator is derived we must digress and review some relevant statistical theory.

\subsection{Efficient estimation of slope parameters}

In this subsection we review the statistical principles used to derive the semiparametric efficient estimators we use in this paper for analyzing productive efficiency. Statistically efficient estimation in semiparametric models is discussed at length in Begun et al. (1983), and Bickel et al. (1993). Newey (1990) and Pagan and Ullah (1999) provide excellent and relatively accessible surveys. The basic ideas are somewhat intuitive. Let $(X, Y)$ stand for a model's generic observations on the exogenous and endogenous variables and let $\mathcal{P}$ be the set of all possible joint distributions of $(X, Y)$. In the semiparametric model there are parameters of interest (e.g., the slope parameters) and parameters that are of no direct interest and are referred to as nuisance parameters (e.g., the distribution of the effects in a panel frontier model). Partition the parameters of the model $(\theta)$ into those of interest $(\beta)$ and those referred to as nuisance parameters $(\eta)$ so that $\theta=\left(\beta^{\prime}, \eta^{\prime}\right)^{\prime}$. Let $\mathcal{P}_{0}$ be a regular parametric submodel (see Ibragimov and Has'minskii, 1981, Section 1.7) and let $P\left(=P_{\left(\beta_{0}, \eta_{0}\right)}\right)$ belong to it. Let $\ell(X, Y, \beta, \eta)$ denote the log likelihood of an observation from $P_{(\beta, \eta)}$ and let the scores with respect to the parameters of interest and the nuisance parameters be $\ell_{\beta}(X, Y)=\partial \ell /\left.\partial \beta\right|_{\left(\beta_{0}, \eta_{0}\right)}$ and $\ell_{\eta_{j}}(X, Y)=\partial \ell /\left.\partial \eta_{j}\right|_{\left(\beta_{0}, \eta_{0}\right)}$,respectively, where $\eta=\left(\eta_{1}, \ldots, \eta_{k}\right)$. Define the efficient score function as $\ell^{*}=\ell_{\beta}-\pi\left(\ell_{\beta} \mid\left[\ell_{\eta}\right]\right)$. The vector

$\left[\ell_{\eta}\right]$ simply denotes the linear span $(S)$ generated by $\left\{\ell_{\eta_{j}}\right\}_{j=1}^{k}$, and $\pi(\ell \mid S)$ denotes the vector of projections of each component of $\ell$ onto the space $S$ using the $L_{2}$ norm. The scores with respect to the parameters of interest are projected onto the nuisance parameter tangent space and then the scores are purged of these projections to get the efficient scores. They are thus designed in such a way as to be orthogonal to information contained in set of nuisance parameters. Such an estimator of the parameters of interest is adaptively estimable (Pagan and Ullah, 1999, p. 218 ) in that it does not require knowledge of the nuisance parameters but is still efficient. The estimator of $\beta$ is called semiparametric efficient if it is asymptotically normal with mean $\beta$ and variance $N^{-1} I^{-1}(P ; \beta)$ where $I(P ; \beta)=E \ell^{*} \ell^{*^{\prime}}$ is the information matrix for the semiparametric 
estimator of $\beta$. The asymptotic distribution of the semiparametric efficient panel estimator $\widehat{\beta}_{N, T}$ is

$$
\sqrt{N T}\left(\widehat{\beta}_{N, T}-\beta\right) \rightarrow N\left(0, I^{-1}(P ; \beta)\right) .
$$

A method of finding $I(P ; \beta)$ is discussed in Bickel et al. (1993). Suffice it to say that for the models we discuss the derivations are often less than straightforward.

\subsection{Estimation of individual effects and the level of the frontier function}

Given the semiparametric efficient estimator $\widehat{\beta}_{N, T}$ it is natural to predict the individual efficiency effects $\alpha_{i}$ by the within residuals $S_{i}\left(\widehat{\beta}_{N, T}\right)$

$$
\hat{\alpha}_{i}=\bar{S}_{i}\left(\widehat{\beta}_{N, T}\right)
$$

With fixed T Park et al. (PSS, 1998) show that

$$
\hat{\alpha}_{i}-\alpha_{i}=O_{p}\left(N^{-1 / 2}\right) .
$$

With $T \rightarrow \infty$ and $N$ fixed or tending to infinity PSS also show that

$$
\sqrt{T}\left(\hat{\alpha}_{i}-\alpha_{i}\right) \rightarrow N\left(0, \sigma^{2}\right) .
$$

The relative technical inefficiency of the $i$-th firm with respect to the $j$-th firm is specified by the difference $\left(\alpha_{i}-\alpha_{j}\right)$. This can be estimated by $\hat{\alpha}_{i}-\hat{\alpha}_{j}$ which has the asymptotic $N\left(0,2 \sigma^{2}\right)$ distribution when normalized by $\sqrt{T}$ under the same assumptions as those used in Theorems 3.4 and 4.1 of PSS (1998).

The support of the marginal distribution of the effects $a_{i}$ is the upper (lower) boundary $B$. A natural estimator of this quantity is

$$
\hat{B}=\max _{1 \leq i \leq N} \bar{S}_{i}\left(\widehat{\beta}_{N, T}\right)
$$

This fact was pointed out by Greene (1980) and utilized by Schmidt and Sickles (1984) and Cornwell et al. (1990) in developments of their panel stochastic frontier estimators. Theorems 4.2 and 4.3 of PSS (1998) are useful in specifying the asymptotic properties of the estimator of the boundary of the support. Let $\alpha_{(N)}=\max _{1 \leq i \leq N} \alpha_{i}$. Then under a set of mild regularity conditions as $T \rightarrow \infty$ the following can be proven:

1.For fixed $N$,

$$
\sqrt{T}\left(\hat{B}-\alpha_{(N)}\right)=O_{p}(1) .
$$

2.For large $N$,

$$
\sqrt{T}\left(\hat{B}-\alpha_{(N)}\right)=O_{p}(\log N)
$$

3.For large $N$,

$$
\alpha_{(N)}-B=O_{p}\left(N^{-\frac{1}{\delta+1}}\right) .
$$

Here the choice $\delta$ determines the extent to which the marginal density of the effects has a certain nonnegligable mass near the boundary point $B$. When $\delta=0$, which would be the 
case where the density at the boundary stays away from zero (such as a shifted half-normal or exponential), then

$$
\hat{B}-B=O_{p}\left(T^{-1 / 2} \log N+N^{-1}\right)
$$

if both $N$ and $T$ go to infinity.

\subsection{The PSS SPE Estimators}

The models for which the SPE estimators have been derived vary depending on how the basic model assumptions are modified to accommodate a particular issue of misspecification of the underlying efficiency model. In the Monte Carlo simulations detailed in section 4, we consider a number of SPE estimators that differ on the basis of assumed orthogonality of effects and regressors, temporal variation in the efficiency effects, and correlation structure of the population disturbance. These estimators are based on the series of papers by Park and Simar (1994) and Park et al. (1998, 2003a, b) and are all based on principles discussed in the previous subsection. The interested reader can find the technical derivations of these estimators therein. These dependency structures and the other forms of potential misspecification have been addressed in the parametric estimation literature in a number of studies surveyed in Baltagi (1995).

The derivations of the different SPE estimators used in the Monte Carlo simulations are based on different parametric submodels that are consistent with the underlying assumptions being addressed in the various modeling scenarios. The parametric submodels are used to derive estimators along the lines described in Section 2.1 For example, when one believes the effects and all of the regressors are dependent and is unwilling to specify a parametric distribution for the dependency structure then one can specify the joint distribution using kernel smoothers. With the joint distribution specified as $h(\cdot, \cdot)$ PSS (1998) showed that the SPE efficiency estimator is the familiar within efficiency estimator introduced by Schmidt and Sickles (1984). Implementation of this estimator (and its variants below) utilize kernel functions $K$ with bandwidth parameter(s) $s_{N}$ which tend to zero at certain rates. When there is no correlation between the effects and the regressors then the within estimator is no longer semiparametric efficient. In this case the joint distribution of the effects and regressors is $h(\cdot, \cdot)=h_{1}(\alpha) h_{2}(X)$. The semiparametric efficient estimator for this pure random effects model is derived in Park and Simar (1994). When there is correlation between the effects and a subset of $q$ regressors $X_{i t}^{(2)}$ where $X=\left[X^{(1)}, X^{(2)}\right]$ then we can assume that $\alpha_{i}$ and $X_{i t}^{(1)}$ are conditionally independent in which case the joint density of the effects and the regressors can be written as

$$
h\left(\alpha, X^{(1)}, X^{(2)}\right)=h_{1}\left(\alpha, X^{(2)}\right) h_{2}\left(X^{(1)} \mid X^{(2)}\right) .
$$

A variant of this model is one in which the dependence between $\alpha$ and $X^{(2)}$ is through long run levels of $X^{(2)}$. In the stochastic frontier production function setting this sort of dependency between $\alpha$ and long run levels in $X^{(2)}$, proxied by $\overline{X^{(2)}}$, may be a natural result of misapplication of technology accompanied by long run changes in factors which contribute to technology's

misapplication. Although one can allow for general dependencies between the effects and the 
regressors with which they are correlated, the semiparametric estimator that results is based on a $(T q+1)$-dimensional kernel estimator whose convergence rate (for fixed $T$ ) is quite slow unless both $T$ and $q$ are small. However, if the joint density of $\left(\alpha, X^{(2)}\right)$ is restricted to be

$$
h_{1}\left(\alpha, X^{(2)}\right)=h_{3}\left(\alpha, \overline{X^{(2)}}\right) h_{4}\left(X^{(2)}\right) \text {, }
$$

then the dimensionality of the joint distribution is reduced to a $(q+1)$-dimensional density. For cases in which $q$ is small this estimator will have relatively rapid convergence properties. This is the Model 3 used in the PSS (1998) analysis of efficiency differences in European and U. S. airline carriers in which differences in long run labor requirements $(q=1)$ were allowed to be correlated with airline technical efficiencies.

Misspecification of production dynamics is a basic problem that is seldom addressed in stochastic frontier models of inefficiency (Ahn, et al., 1998, 2000, Alam, and Sickles, 2000). A semiparametric efficient estimator of the stochastic production function that allows for such misspecified dynamics in the guise of an $A R(1)$ population disturbance term is derived in PSS (2003a). In this case the error term in Eq. (1) is written as

$$
\varepsilon_{i t}=\rho \varepsilon_{i, t-1}+u_{i t} ; \quad|\rho|<1 \text {. }
$$

Different dependency structures between $X$ and $\alpha$ also can be considered for the $A R(1)$ model, as with the models of PSS (1998, 2003a). Construction of the semiparametric efficient estimator under different dependency structures utilizes kernel smoothers for the nonparametrically specified distribution of the effects and the regressors.

\section{Alternatives to the Semiparametric Efficient Estimators}

There are a number of other panel frontier estimators that have been used widely and/or largely ignored in the empirical efficiency literature. They differ from the SPE estimators in terms of the assumptions made about the distribution of the unobserved efficiency effects and about the correlation between the efficiency effects and the regressors.

Cornwell et al. (CSS) (1990) introduced a set of generalized least squares and instrumental variables (IV) estimators that allow for cross-sectional and temporal heterogeneity in efficiency, correlation of efficiency effects with all or some of the regressors, and identification of coefficients of time invariant regressors. Although their estimators were first used to measure time variant firm efficiencies they have appeal in generic panel data problems as well. The model they consider generalizes (1) by specifying the production function as

$$
Y_{i t}=X_{i t}^{\prime} \beta+Z_{i}^{\prime} \gamma+W_{i t}^{\prime} \delta_{i}+\varepsilon_{i t},
$$

where $Z_{i}$ is a vector of $(J)$ time invariant regressors, $W_{i t}$ is vector of $(L)$ regressors whose coefficients vary with the cross-sectional unit and can display both cross-sectional and time series variation. If $W$ contains just a constant term then this model reduces to Eq. (1), albeit with an expanded set of $J$ regressors. Let $\delta_{i}=\delta_{0}+u_{i}$. Then we can rewrite the model as 


$$
\begin{aligned}
& y_{i t}=X_{i t}^{\prime} \beta+Z_{i}^{\prime} \gamma+W_{i t}^{\prime} \delta_{0}+v_{i t}, \\
& v_{i t}=W_{i t}^{\prime} u_{i}+\varepsilon_{i t} .
\end{aligned}
$$

The $u_{i}$ are assumed to be iid zero mean random variables with covariance matrix $\Delta$. The disturbances $\varepsilon_{i t}$ are taken to be iid with a zero mean and constant variance $\sigma^{2}$, and are uncorrelated with the regressors and $u_{i}$.

It is convenient to work with the matrix version of model. This is given by

$$
\begin{aligned}
& y=X \beta+Z \gamma+W \delta_{0}+v, \\
& v=Q u+\varepsilon,
\end{aligned}
$$

where $W$ is $N T \times L, Q=\operatorname{diag}\left(W_{i}\right), i=1, \ldots, N$, is $N T \times N L$, and $u$ is $N L \times 1$.

We assume that $L \leq T$, so that $Q$ is of full column rank. Although this is not necessary to identify $\beta$ it is necessary to identify the individual $\delta_{i}$. Taking $Q$ to be of full column rank, let $P_{Q}=Q\left(Q^{\prime} Q\right)^{-1} Q^{\prime}$ be the projection into the column space of $Q$ and $M_{Q}=I-P_{Q}$ be the projection onto the null space of $Q$. Below we consider a number of estimators for this model.

\subsubsection{The Parametric Within Estimator}

The generalization of the standard within estimator for a model with heterogeneity in slopes as well as in intercepts is based on the transformation of the model via $M_{Q}$ after which we simply run least squares. Since $M_{Q} Z=0, \gamma$ cannot be estimated. The within estimator of $\beta$ is given by

$$
\widehat{\beta}_{w}=\left(X^{\prime} M_{Q} X\right)^{-1} X^{\prime} M_{Q} y .
$$

The within estimator is an IV estimator with instruments $M_{Q}$ (or equivalently $M_{Q} X$ ). Its consistency does not depend on assumptions of uncorrelatedness of $(X, Z)$ and $Q u$.

\subsubsection{The Parametric Generalized Least Squares Estimator}

The GLS estimator is ordinary least squares applied to the transformed equation

$$
\Omega^{-1 / 2} y=\Omega^{-1 / 2} X \beta+\Omega^{-1 / 2} Z \gamma+\Omega^{-1 / 2} W \delta_{0}+\Omega^{-1 / 2} v,
$$

where it can be shown that

$$
\Omega^{-1 / 2}=\frac{1}{\sigma} M_{Q}+F
$$

and

$$
F=Q\left(Q^{\prime} Q\right)^{-1 / 2}\left[\sigma^{2} I_{N L}+\left(Q^{\prime} Q\right)^{1 / 2}\left(I_{N} \otimes \Delta\right)\left(Q^{\prime} Q\right)^{1 / 2}\right]^{-1 / 2}\left(Q^{\prime} Q\right)^{-1 / 2} Q^{\prime} .
$$

The consistency of GLS hinges on the uncorrelatedness of $(X, Z, W)$ and $Q u$. However, GLS

allows the estimation of $\gamma$, and for fixed $T$, it is more efficient than the within estimator. The feasible GLS estimator is based on consistent estimates of $\Omega^{-1 / 2}$ which are given in CSS. 


\subsubsection{The Extended Parametric Hausman-Taylor (H-T) Estimator}

Now consider the case in which some of the regressors are correlated with the effects. In particular assume that $B=\left(X_{1}, Z_{1}, W_{1}\right)$ are uncorrelated with the effects in the sense that $\operatorname{plim}(N T)^{-1} X_{1}^{\prime} Q u=0$, and similarly for $Z_{1}$ and $W_{1}$, while $\left(X_{2}, Z_{2}, W_{2}\right)$ are correlated with the effects. Let the dimensions of $X_{1}, Z_{1}, W_{1}, X_{2}, Z_{2}, W_{2}$ be $k_{1}, j_{1}, l_{1}, k_{2}, j_{2}, l_{2}$ (with $k_{1}+k_{2}=K$, $j_{1}+j_{2}=J$, and $\left.l_{1}+l_{2}=L\right)$.

A generalization of the $\mathrm{H}-\mathrm{T}$ simple, consistent estimator is obtained by first using the within estimator and constructing the within residuals as

$$
\left(y-x \widehat{\beta}_{w}\right)=Z \gamma+W \delta_{0}+\left[Q u+\varepsilon+X\left(\beta-\widehat{\beta}_{w}\right)\right] .
$$

We transform this equation by premultiplying by $\Omega^{-1 / 2}$

$$
\Omega^{-1 / 2}\left(y-x \widehat{\beta}_{w}\right)=\Omega^{-1 / 2} Z \gamma+\Omega^{-1 / 2} W \delta_{0}+\Omega^{-1 / 2}\left[Q u+\varepsilon+X\left(\beta-\widehat{\beta}_{w}\right)\right] .
$$

The simple consistent estimator is then defined as the instrumental variables estimator of this equation using as instruments

$$
B^{*}=\Omega^{-1 / 2} B=\Omega^{-1 / 2}\left(X_{1}, Z_{1}, W_{1}\right) .
$$

Note that $B$ is transformed by $\Omega^{-1 / 2}$. The use of untransformed instruments is clearly suboptimal, if we assume "reduced form" equations for $\left(X_{2}, Z_{2}, W_{2}\right)$ which are linear in $\left(X_{1}, Z_{1}, W_{1}\right)$. The simple consistent estimator using the transformed instruments is

$$
\left[\begin{array}{c}
\widehat{\gamma}_{w} \\
\widehat{\delta}_{0 w}
\end{array}\right]=\left[(Z, W)^{\prime} \Omega^{-1 / 2} P_{B^{*}} \Omega^{-1 / 2}(Z, W)\right]^{-1}(Z, W)^{\prime} \Omega^{-1 / 2} P_{B^{*}} \Omega^{-1 / 2}\left(y-x \widehat{\beta}_{w}\right) .
$$

The estimator will exist if we have enough instruments, i.e. if $k_{1}+j_{1}+l_{1} \geq J+L$, or equivalently if $k_{1} \geq j_{2}+l_{2}$.

The efficient IV estimator estimates the equation

$$
\Omega^{-1 / 2} y=\Omega^{-1 / 2} X \beta+\Omega^{-1 / 2} Z \gamma+\Omega^{-1 / 2} W \delta_{0}+\Omega^{-1 / 2} v
$$

by IV, using as instruments $A^{*}=\Omega^{-1 / 2} A=\Omega^{-1 / 2}\left(M_{Q}, X_{1}, Z_{1}, W_{1}\right)$. Let $G=(X, Z, W)$. Then the efficient IV estimator is

$$
\left[\begin{array}{c}
\widetilde{\beta}^{*} \\
\widetilde{\gamma}^{*} \\
\widetilde{\delta}_{0}^{*}
\end{array}\right]=\left(G^{\prime} \Omega^{-1 / 2} P_{A^{*}} \Omega^{-1 / 2} G\right)^{-1} G^{\prime} \Omega^{-1 / 2} P_{A^{*}} \Omega^{-1 / 2} y .
$$

Conditions for the existence of this estimator and the relationship between the efficient and simple consistent estimators can be found in CSS.

A remaining issue is how to consistently (for large $N$ ) estimate $\Omega$ whose unknown parameters are $\sigma^{2}$ and $\Delta$. If $S S E_{w}$ is the unexplained sum of squares in the within regression then $\widehat{\sigma}^{2}=S S E_{w} / N(T-1)$ is a consistent estimator of $\sigma^{2}$. To estimate $\Delta$, let $\mathrm{e}_{i}$ be the IV residuals for cross-section i (e.g., from the simple consistent IV estimator) and define 


$$
\widehat{\Delta}=\frac{1}{N} \sum_{i=1}^{N}\left[\left(W_{i}^{\prime} W_{i}\right)^{-1} W_{i}^{\prime} e_{i} e_{i}^{\prime} W_{i}\left(W_{i}^{\prime} W_{i}\right)^{-1}-\widehat{\sigma}^{2}\left(W_{i}^{\prime} W_{i}\right)^{-1}\right],
$$

it can be shown that this is a consistent estimator (for large $N$ ) of $\Delta$.

\subsection{A Generalization of Parametric Models of Efficiency: The Mixed Effect Model}

Cornwell et al. (1990) relaxed the assumption that the effects are time-invariant by replacing the firm effect $\alpha_{i}$ with a flexibly parameterized function of time, with parameters that vary over firms. The parametrization chosen in CSS was a quadratic function of time $\alpha_{i t}=\theta_{i 1}+\theta_{i 2} t+\theta_{i 3} t^{2}$. Other than a quadratic function of time, a random effects specification of $\alpha_{i t}$ has been modeled as $\alpha_{i t}=\gamma(t) \alpha_{i}=\left[1+\exp \left(b t+c t^{2}\right)\right]^{-1} \alpha_{i}$ (Kumbhakar, 1990), and $\alpha_{i t}=\eta_{i t} \alpha_{i}=\exp [-\eta(t-T)] \alpha_{i}$ (Battese and Coelli, 1992), and $\alpha_{i t}=\theta_{t} \alpha_{i}$ (Lee and Schmidt, 1993), all of whom used mle to estimate efficiency.

Kneip (1994) proposed an alternative to the fixed effect estimator that allowed for a very general heterogeneity structure and relied on principal components. This approach is more general than fitting polynomials and can be used to parsimoniously model virtually any temporal pattern of firm efficiency. To measure time -variant heterogeneity (1) can be estimated using a two-step procedure. In the first step, the model is estimated to obtain consistent estimates of $\beta$. In the second step, principal components are applied to the residual $\varepsilon_{i t}=\alpha_{i t}+v_{i t}$ with

$$
\alpha_{i t}=c_{i 1} g_{1 t}+c_{i 2} g_{2 t}+\cdots+c_{i L} g_{L t}
$$

where $c_{i r}$ are unknown parameters, and $g_{i r}$ are smooth, real-valued functions. The $g_{i r}$ 's are referred to as basis functions. The smallest $L$ such that the model holds for some appropriate set of basis functions is the dimension of the model. In order to operationalize this approach to efficiency measurement, it is necessary to estimate the parameters $c_{i r}$, the functions $g_{i r}$, and the number $(L)$ of $g_{i r}$ functions. The firm efficiencies are obtained from the structures of the $g_{i r}$ and from the distribution of the effects $\alpha_{i}$. The fixed effect model is nested in the mixed efficiency effects specification $\left(L=1, g_{r t}=1, c_{i r}=\gamma_{i}\right.$ for all $r$ and $\left.t\right)$. Methods for estimating $c_{i r}, g_{i r}$, and $L$ are found in Kneip (1994) and Kneip et al. (2003).

\subsection{Parametric Model of Efficiency and of Its Temporal Variation-The Bat- tese and Coelli Estimator}

We also analyze efficiencies using the fully parameterized maximum likelihood estimator introduced by Battese and Coelli (1992). This estimator models technical efficiency as an exponential function of time. The panel frontier is modified somewhat by specifying $\alpha_{i t}=\eta_{i t} \alpha_{i}=$ $(\exp [-\eta(t-T)]) \alpha_{i}$. The $\epsilon_{i t}$ are assumed to be iid $\mathrm{N}\left(0, \sigma_{V}^{2}\right)$ random errors while the $\alpha_{i}$ are iid nonnegative truncations of an $\mathrm{N}\left(0, \sigma^{2}\right) ; \eta$ is an unknown scalar parameter.

Note that the variation of the firm effects depends on the parameter $\eta$. The effects decrease, remain constant, or increase as $t$ increases, if $\eta>0, \eta=0$, or $\eta<0$. One obtains the standard time invariant technical efficiency measure when $\eta=0$. 


\subsection{Nonparametric Methods to Estimate Technical Efficiency}

Shephard's (1953) input distance function establishes a formal linkage between the observed technology and technical efficiency. Estimation of technology can be based on a parametric functional form (e.g., the Cobb-Douglas or translog) or a nonparametric representation of technology. One such nonparametric representation of technology can be implemented through linear programming methods which are powerful, easy, and impose minimal assumptions on the boundary of the input requirements set. The assumptions include piece-wise linearity and convexity. The technology can be represented by the input distance function, which involves scaling the input vector $x$ :

$$
D(y, x)=\max \left\{\theta:\left[\frac{1}{\theta}\right] x \in L(y)\right\}
$$

where $L(y)$ is the input requirement set, which is a set of input vectors $x$ that can produce the output vector $y$, and $\theta \geq 1$. Clearly, $D(y, x) \geq 1$ and is a measure of technical efficiency since the isoquant is the set of observations on $x$ that are on the boundary of the input requirement set at which $D(y, x)=1$. The Debreu (1951) and Farrell (1957) input-based measure of technical efficiency is

$$
T E(y, x)=\min \{\theta: \theta x \in L(y)\},
$$

where $0 \leq \theta \leq 1$. It is clear that $T E(y, x) \leq 1$ and that $T E(y, x)=1 / D(y, x)$. We can similarly define output-oriented distance functions.

These distance functions can be calculated using linear programming methods that have become known generically as data envelopment analysis (DEA). Charnes et al. (1978), Banker et al. (1984) and Färe et al. (1985, 1994) develop and refine these linear programming approaches to measure technical efficiency. For the input-oriented case, the DEA linear program for the intertemporal production set is

$$
\begin{aligned}
D_{i t}\left(y_{i t}, x_{i t}\right) & =\min _{\theta, \lambda} \theta \\
\text { s.t. }-y_{i t}+Y \lambda & \geq 0, \\
\theta x_{i t}-X \lambda & \geq 0, \\
\lambda & \geq 0,
\end{aligned}
$$

where $\theta$ is a scalar, $\lambda$ is an $(N T \times 1)$ vector of constants and $i=1 \ldots N ; t=1, \ldots, T$. The efficiency score of the $i$ th firm at time $t$ is given by $0 \leq \theta \leq 1$, where 1 indicates a point on the frontier and hence a technically efficient firm. We solve $N T$ linear programs to obtain a value of $\theta$. Essentially, this linear program radially contracts the input vector of the $i t h$ firm, $x_{i}$, to a projected point $(X \lambda, Y \lambda)$, on the surface of the piece-wise linear isoquant or technology. ${ }^{2}$

\footnotetext{
${ }^{2}$ The sampling variation of DEA-based efficiency estimators also can be constructed by recently developed bootstrap procedures introduced by Simar and Wilson $(1999,2000)$ who outline a smooth bootstrap procedure to examine the sensitivity of distance functions, and hence efficiency, to sampling variation of the frontier. We do not pursue this in our experiments below.
} 


\section{Monte Carlo Experimental Results}

We have discussed a number of competing estimators of $\beta$ and $\alpha_{i}$. In this section we examine the finite sample performances of the estimators through the following Monte Carlo (MC) scenarios.

The basic model we simulate is Eq. (1). We simulate samples of size $N=10,50$ with $T=10,30,50$ in a model with $d=2$ regressors. In each MC sample, the regressors were generated independently according to a bivariate VAR model

$$
X_{i t}=R X_{i, t-1}+\eta_{i t}, \text { where } \eta_{i t} \sim N_{2}\left(0, \sigma_{X}^{2} I_{2}\right),
$$

where $\sigma_{X}=1$ and $R=\left(\begin{array}{cc}4 & 0.05 \\ 0.05 & .4\end{array}\right)$.

The simulation was initialized by choosing $X_{i 1} \sim N_{2}\left(0, \sigma_{X}^{2}\left(I_{2}-R^{2}\right)^{-1}\right)$ and then starting iteration (15) for $t \geq 2$.

The resulting values of $X_{i t}$ then were shifted around three different means to obtain 3 balanced groups of firms from smaller to larger. We fix $\mu_{1}=\left(\begin{array}{ll}5 & 5\end{array}\right)^{\prime}, \mu_{2}=\left(\begin{array}{ll}7.5 & 7.5\end{array}\right)^{\prime}, \mu_{3}=\left(\begin{array}{ll}10 & 10\end{array}\right)^{\prime}$. The idea is to generate a reasonable cloud of points for $X$. Other scenarios have been tried: they influence the quality of the estimators jointly but they do not change the conclusions on the comparison issue raised here.

For the experiments in which efficiency was not varying over time and cross-section, the inefficiency parts (the individual effects) were generated as $B-\operatorname{Expo}\left(\mu_{\alpha}\right)$ where we chose for the exponential distribution (denoted by $\operatorname{Expo}(\cdot)$ ) a mean $\mu_{\alpha}=1$ and for the upper boundary a value of $B=1$. Since the $Y^{\prime} s$ and $X^{\prime} s$ are measured in logarithms this involves an average inefficiency score of $50 \%\left(E\left(\exp \left\{-\operatorname{Expo}\left(\mu_{\alpha}\right)\right\}\right)=0.50\right)$. For the experiments in which efficiency was changing over time we modified the generation of the effects to mirror the assumed temporal patterns of an $A R M A(0.9,0.9)$ process. For the experiments in which the regressors were allowed to be correlated with the effects we generated the draws for $\eta_{i t}$ (the stochastic portion of $X_{i t}$ ) and the $\alpha_{i}^{\prime} s$ from a bivariate process with correlation set at 0.5 to reflect a nominal level of correlation. Other scenarios for generating the $\left(X_{i t}, \alpha_{i}\right)$ could be chosen but this does not affect the conclusions below. The values of $\beta$ were set equal to $\left(\begin{array}{ll}0.5 & 0.5\end{array}\right)^{\prime}$ to represent a constant returns to scale technology. The disturbances are drawn from a normal distribution with $\sigma=0.5$. For the experiments in which the disturbances follow an $A R(1)$ process the autoregressive part was generated with $\rho=0.8$ and $\sigma=0.5$. Changing the value of $\sigma$ affects jointly the quality of the estimators but not the comparisons below. The number of Monte Carlo simulations was $(M)=100 .^{3}$

\footnotetext{
${ }^{3}$ Programs are written in Matlab 6.5. The simulations are very computationally challenging. For N=100, $\mathrm{T}=50$, and $\mathrm{N}=500$ one experiment takes approximately $80 \mathrm{~h}$ on a Pentium III processor running at $1.8 \mathrm{GHz}$. Presumably these could be speeded up by customizing the programs to run on parallel machines and/or by utilizing faster processors than those available to us. Because of the severe computational burden of these experiments we have had to cut back the number of Monte Carlo simulations from 500 to 100 and the largest cross-sectional sample sizes from 100 to 50. We have examined results from several different experimental setups based on these different numbers of simulations and cross-sectional sizes and have found no qualitative differences and little quantitiative difference in our results.
} 
Since the VAR process generating the regressors $X_{i}$ is symmetric in both components, the $M S E$ for the estimators of the two coefficients of technology are of the same order of magnitude. In the first set of tables, we display the sum of the two MC mean squared errors:

$$
M S E=\sum_{j=1}^{2} \frac{1}{M} \sum_{m=1}^{M}\left(\hat{\beta}_{j}^{m}-\beta_{j}\right)^{2},
$$

where $\hat{\beta}$ is based on one of the competing estimators. Estimates of the fixed/random efficiency effects and of relative efficiencies are analyzed by computing correlations and rank correlations between the true effects and those based on the different estimators. Comparability of the different efficiency estimators is gauged by examining the correlations of level effects and of relative efficiencies among the estimators. For the bandwidth $s$ we selected an optimal fixed value $s^{*}$ by running the whole Monte Carlo experiment for a selected grid of 20 equally spaced values for $s$ between 0.1 to 2 . We report in the tables the results corresponding to the optimal bandwidth $s^{*}$ which minimizes the MSE. In all the tried scenarios, the results were not highly sensitive to the choice of $s$ in the above grid.

We begin with a rather benign data generating process in which there exist no problems: the disturbance is iid, the effects are constant and generated from a lognormal distribution (note that the BC estimator assumes that the effects are half-normal), there is no correlation between the effects and the regressors, and there are no misspecified dynamics. We refer to this experimental setup as the "no problems" setup. We then present experimental results for cases in which the disturbance exhibits serial correlation $(\rho=0.8)$, regressors are correlated with efficiency (correlation between regressor and efficiency is 0.5 for both regressors), the efficiencies are temporally variant following the $A R M A(0.9,0.9)$ process descibed above, and when all of these problems exist. The seminonparametric, parametric, and nonparametric estimators we consider are within (Model 1 of PSS, 1998), random effects GLS, Hausman-Taylor (1981), PSS Model 3 (long-run levels of the regressors are correlated with the efficiency effects), the Park et al. (2003a) semiparametric within and GLS estimators when the disturbance is serially correlated (PSSW and PSSG), the CSS within and GLS estimators with quadratic temporal change for each firm's efficiency (CSSW and CSSG), the Battese and Coelli estimator (B-C), the Kneip et al. (2003) mixed effects estimator (KSS), and the nonparametric linear programming method referred to as DEA. Results for the sum of the mean-squared-errors (mse) of the two parameter estimates and of the mse of the efficiency estimates (averaged over the simulations) are found in Tables 1-10. Results for the correlations between true efficiencies (like the other parameters of the model they are fixed for a particular MC experiment) and the estimated efficiencies are averaged over the simulations and are found in Tables 11-18 for selected experiments.

We first discuss results when efficiency is temporally invariant, the erros are not serially correlated, and there is no correlation between the regressors and technical efficiency. The mse of estimators and of technical efficiencies are Tables 1 and 2. For this dgp the methods have very little to distinguish among themselves. That is a good thing for researchers who are uncertain as to which of the many alternative estimators they should use with panel data. Technology is measured very accurately by all estimators with relative mse's favoring no particular estimator in regard to estimates of the $\beta^{\prime} s$. The $N, T$ asymptotics of each estimator is revealed in the pattern 
of the mse's as both $N$ and $T$ are increased. The design of the experiments did not address issues of relative time invariance of the regressors. Thus, estimators that utilize some variant of the within transformation (Within, H-T, PSS1, CSSW) may overstate their relative performance when regressors do have such patterns. Estimators such as CSSW, CSSG, B-C, KSS that parameterize time varying efficiencies when in these experiments they are constant, suffer in both mse measures. DEA also suffers relative to the other estimators in regard to technical efficiency measurement. However, these results are the most favorable to the estimators that are designed for this dgp, in particular the GLS-based and the B-C estimators that model efficiency as a random effect. Correlations between the true and estimated efficiencies are quite high $(>0.95)$ for all these estimators for all configurations of cross-section and time series observations and, not surprisingly, quite high among themselves.

We next introduce misspecified dynamics in the form of a serially correlated error term. The results for mse's of the $\beta^{\prime} s$ and for the technical efficiencies are in Tables 3 and 4 . This experimental setting is one in which the PSS2 estimators should be at an advantage and they clearly are, with mse's about $50 \%$ smaller than for the other estimators that either ignore serial correlation, mistake it for a portion of the serial correlation structure inherited from their random effects assumptions, or mistake it as temporally varying efficiency when no temporal variation in technical efficiency exists. The mse of the estimators of $\beta$ have increased five-fold over those in Tables 1 and 2 for most of the $N, T$ and estimator combinations. As information increases in the form of a larger cross-section to identify the parameters and a larger time series to identify the correlations in the disturbances, most estimators again converge to similar mse's, with GLSbased estimators holding a slight edge over those that do not deal formally with any nonstandard correlation pattern such as that found in the composed error term. The mse's for estimators of technical efficiency increase by almost an order of magnitude for the largest samples $(N, T=50)$, although we now see a pattern emerging in which estimators that allow for temporal variation in efficiency (CSSW-CSSG,B-C,KSS) appear to do relatively better than when no dynamics were specified. The DEA estimator's mse deteriorates somewhat with this form of misspecification and is comparable with the KSS estimator in terms of the mse of technical efficiency relative to the other estimators for the largest $N, T$ samples. Tables 11 and 12 provide both standard and Spearman rank correlations between the true and estimated technical efficiencies. Here the correlations are relatively high over most of the estimators and $N, T$ combinations, although the KSS estimator, in particular, performs poorly when efficiency is not temporally varying and when there is simply serial correlation in the disturbance. The other estimators, however, provide technical efficiency estimates that are highly correlated with the true efficiency. The correlations increase for larger $T$ (fixed $N$ ) and for larger $N$ (fixed $T$ ).

Correlation between technical efficiency and regressors is a natural extension of the efficiency model. Technical efficiency may be due in part to changes in a particular regressor that determines output, e.g., the sluggish adjustment of a quasi-fixed factor such as labor in European national airlines before liberalization efforts in the late 1990s (Park et al., 1998). Tables 5 and 6 report Monte Carlo results when such correlations (0.5) exist between the efficiency terms and the two regressors in our experiments. This form of misspecification does not appear to compromise the ability of estimators to track either the coefficients or the technical efficiencies 
relative to the case in which no correlation existed. Nor does there appear to be any clear distinction between estimators when correlations between the true and estimated efficiencies are examined - the correlations are uniformly high and we do not report them here. Whether these findings are due to the relatively poor instruments used by consistent IV estimators such as within, H-T, PSS1, CSSW, or due to the relatively small sample sizes used in our analysis is unclear and requires further study.

We now move to an experimental setting in which efficiencies are changing over time for the firms. That different firms have heterogeneous patterns of efficiency is a quite reasonable possibility to consider in modeling efficiency with panel data. A simple time trend common to all firms may oversmooth the substantial heterogeneity in firm-specific temporal adjustments to the best-practice technology. The model for temporal variation was picked to favor no estimator - no estimator we consider is designed to identify and estimate an $A R M A(1,1)$ model with parameters $(0.9,0.9)$. Tables 7 and 8 provide the mse's of estimators of the $\beta^{\prime} s$ and of the technical efficiencies. What begins to emerge from these experiments is that none of the estimators we have considered differs very much in either table with the exception of the KSS estimator. The KSS estimator clearly dominates in regard to the mse measures of Tables 7 and 8. The other estimators do not distinguish themselves in any particular regard. Tables 13 and 14 provide correlations between the true and estimated technical efficiencies. As the number of cross-sections and time series increase it is clear that the KSS and the DEA estimators provided a better correlation between true and estimated technical efficiencies, relative to the other estimators considered. There is no apparent pattern to the comparability of the other estimators, although the CSSW and CSSG appear to do best in terms of Spearman rank correlations for large samples $(N=50, T=50)$. The differences may not be statistically significant. ${ }^{4}$

The last set of experiments uses a dgp based on all of the problems we have examined so far: the disturbance is serially correlated $(\rho=0.8)$, regressors are correlated with technical efficiency $(\rho=0.5)$, and technical efficiency follows an $A R M A(1,1)$. Tables 9 and 10 report the mse of the $\beta^{\prime} s$ and of the estimators of technical efficiency under this experimental setup. Again, the different estimators have relatively little to distinguish themselves other than the KSS estimator that over parameterizes time variation in efficiency (see Tables 1 and 2 when no temporal variation in efficiency exists). Interestingly, when we examine the level and rank correlations between true and estimated efficiencies in Tables 15 and 16, not only does the KSS estimator perform relatively well but so does the DEA estimator. Tables 17 and 18 provide correlations between the different estimators for a particular experiment in this class of experiments $(N=$ $20, T=50)$.

\section{Empirical Illustration of Alternative Efficiency Estimators}

Here we illustrate the various estimators and their temporal patterns using a subset of 50 unit banks from the U. S. banking data analyzed elsewhere by Adams et al. (1999), Park et al. (2003a), and Fernández et al. (2004).

\footnotetext{
${ }^{4}$ We do not calculate the standard error for these correlations for the experiments.
} 
Since the early 1980s U. S. federal and state regulatory agencies have resorted to less stringent interpretation of banking regulations and adopted less restrictive legislature. The passing of the Reigle-Neal Act in the early 1990s enabled nationwide banking, while the relaxing of unit bank, branch bank and state bank-type legislature has resulted in numerous mergers and failures which have significantly altered the U.S. banking environment. The introduction of interest bearing consumer checking accounts and the phasing out of Regulation Q interest rate ceilings on savings and small denomination time deposits in the early 1980s were among the initial wave of deregulation policies. Money market deposit accounts (structured similar to mutual funds) led not only to a new product line but also to competition from non-bank institutions. For comprehensive discussions of these deregulatory issues and the industry's reactions and adjustments to them, see Berger et al. (1995).

Previous studies of banking productivity and efficiency have relied on three basic methods for productivity and efficiency measurement: linear programming, maximum likelihood, and ordinary least squares or instrumental variable estimation. Berger and Humphrey (1997) provide a general description of these methods. Our focus here is on efficient and robust measurement of productivity and efficiency in a setting in which the regulatory climate has been steadily altered, forcing firms to adjust to a best practice technology using resource allocations that are increasingly unconstrained by financial regulation.

The full data set consists of 2,051 U. S. banks from the first quarter of 1980 through the fourth quarter of 1989. We divide the data set into two subsamples based on differing regulatory environments: limited branching (Limit) and no branching (Unit). These samples contain 1,220 and 831 banks each. The production and cost data were obtained on-line from the Federal Reserve Bank of Chicago. The Report of Condition and Income (Call Report) and the FDIC Summary of Deposits are the primary sources for the U.S. banking data. The panel data set is a comprehensive source of information on operating costs, inputs (including labor, capital and purchased funds), outputs (loans and deposit services), and the regulatory environment of any institution in the U. S. banking industry. Data on over 100 variables were collected from the Call Reports and the FDIC Summary of Deposits.

Labor (LAB) is measured using the number of full time-equivalent employees on the payroll at the end of each quarter. The total value of premises, fixed assets, and capitalized leases are used as a proxy for capital (CAP). Purchased funds (PURF) are measured using the sum of deposits greater than U. S. $\$ 100,000$, foreign debt, federal funds purchased, and liabilities on borrowed money.

The measurement of loan and deposit services is a more complex issue, and two approaches are currently utilized in the U. S. banking literature: the intermediation approach and production approach. The intermediation approach uses the dollar amounts of deposits and outstanding loans as a proxy for deposit and loan services provided by a bank, while the production approach uses the number of outstanding loans and deposits as a measure of banking services produced. The former approach is followed in the data collection and in the modeling method.

The following loan and deposit types are used in this study: real estate loans (RELN), commercial and industrial loans (CILN), installment loans (INLN), and retail time and savings deposits (Deposits). CILN accounts for loans given to businesses, while INLN accounts for 
loans given to individuals to meet medical expenses, vacation expenses, purchase furniture, automobiles, household appliances, and other miscellaneous expenses. RELN accounts for loans secured by real estate.

The price (interest rate) for each of the loan types is obtained by dividing the interest rate and fee income earned by the outstanding loan amount. A composite wage rate is obtained by dividing the total labor expenses by the total number of workers. Price indices for capital and purchased funds are calculated by dividing the expenses incurred for each input by the value of total deposits.

Outputs, inputs and price definitions used in this paper are consistent with those used in previous studies. Bank size (total assets) is highly correlated with the size of a given output, and thus dollar values are used in place of the number of loans or deposits.

The definitions of quantities and prices are less than ideal, but are necessitated due to the absence of explicit price indices. The Call Report and FDIC data are reported in nominal terms, and are converted into real terms using a state-level consumer price index (1983-1984=\$100).

\subsubsection{Estimation Results for US Banks}

We utilize a subsample of 50 unit banks to estimate the various models. We focus on the relatively smaller subsample so that we might illustrate the heterogeneity that exists in both levels and temporal patterns of firm efficiencies pictorially. Our analysis focuses simply on the temporal patterns of technical efficiencies for the various estimators, for their simple average, and for variations that exist across firms for selected estimators. They are summarized in Figures 1-5. Figures 1-4 display the temporal patterns and the substantial heterogeneity that exists among firms over time for the BC, CSSW, CSSG, and the KSS estimators. As one can see, the levels of firm efficiencies are quite heterogenous as are their temporal patterns as we allow for greater flexibility in the parameterization of efficiency change. The BC estimator clearly is quite stable owing to its relatively simple parametric structure, while the CSSW and CSSG are clearly less comparable. The KSS estimator would appear to combine the stability of the BC estimator with the temporal flexibility of the CSSW and CSSG estimators. We also show in Figure 5 the comparative temporal patterns of efficiency for the competing estimators discussed in the previous sections. Although the PSS1 and PSS2 estimators can easily accommodate the generalization utilized in the CSS estimator to allow for flexibility in temporal patterns of firm-specific efficiency we estimate a time invariant specification as we also do for the Schmidt and Sickles random effect and fixed effect estimators and H-T estimators. Finally, we combine the estimators into a composite one by simple averaging in Figure 6 . Although the weighting scheme here is clearly naive it does characterize the efficiency findings from the various estimators in a clear and informative summary figure that indicates a ramping up of efficiency in the early 1980s and a flatting of the efficiency changes in the late 1980s. These results are not surprising considering the fact that the banking industry underwent substantial changes during the 1980s. This is particularly true for the early part of the decade, when some deposit types were deregulated, capital requirements were redefined, and banks began to move into nontraditional banking markets. Also, bank failures increased during 1985-1989. As 
banks became accustomed to the new regulations the pace of efficiency growth slowed as the effects of financial deregulation were captured in the business practices utilized by the successful unit banks.

\section{Concluding Remarks}

In this paper the foundations and properties of an array of estimators of technical efficiency available to the applied researcher have been summarized. The model assumptions under which each of the estimators have been developed also have been presented. Some relatively new but possibly inaccessible semiparametric robust estimators have been discussed. The estimators have been examined in a set of Monte Carlo simulations and an illustration of the estimators has been provided. Simulation results point to the relative merit of different estimators under different circumstances. Which estimator is used depends on the reseacher's understanding of the institutional factors in the market under study. Are constraints borne more or less equally by all firms, are they not? Do they change over time? The analysis herein suggests that answers to these questions at the outset of a productivity study of productivity are no less important than the questions.

Based on the results of this paper I have several recommendations for the applied efficiency modeler. The first is to use the most robust procedure available that also allows for flexibility in the temporal patterns of efficiency but which does not force on these patterns too much parametric smoothness. A clear advantage of the class of semiparametric efficient estimators (generalized using the CSS or the mixed effects general specification of temporal firm-specific efficiency - a generalization not pursued in this paper) is that it is often possible to relax some of the strong parametric assumptions about the distribution and dependency structure of the "reduced form" Solow residual while still preserving statistical efficiency for this class. However, relaxing parametric assumptions comes at the price of instability of the temporal patterns of firm-specific efficiency measures. One way out of this dilemma is to combine the various stochastic frontier and DEA models of efficiency in a portmonteau estimator that would weight the various efficiency measures based on a formal Bayes prior on the amount of allowed variability in efficiency measures and parameter estimates. The Bayesian framework for estimating stochastic frontiers introduced by van den Broeck et al. (1994) and extended in this Annals Issue by Fernández et al. (2004) could be adopted to formalize what applied researchers often informally adopt in their research-downweight estimates of inefficiency that are unduly rough and instable. Currently I am pursuing this strategy and have freeware available that utilizes $e x$ post a uniform prior for weighting the estimators (http://www.ruf.rice.edu/ $\sim$ rsickles/). 


\section{References}

[1] Adams, R. M., and R. C. Sickles (1999), "Semi-parametric efficient distribution free estimation of panel models," Rice University, mimeo.

[2] Adams, R.M., Berger, A.N., and Sickles, R.C. (1997), "Computation and inference in semiparametric efficient estimation," Advances in Computational Economics, edited by Hans Amman, Berc Rustem and Andrew Whinston, Boston: Kluwer Academic, 57-70.

[3] Adams, R.M., Berger, A.N., and Sickles, R.C. (1999), "Semiparametric approaches to stochastic panel frontiers with applications in the banking industry", Journal of Business and Economic Statistics, 17, 349-358.

[4] Afriat, S. (1972), "Efficiency estimation of a production function," International Economic Review, 13, 568-98.

[5] Ahn, S, Good, D., and R. C. Sickles (1998), "Assessing the relative efficiency of Asian and North American airline firms," Economic Efficiency and Productivity Growth in the Asia Pacific Region, Chapter 4, Tsu-tan Fu, Cliff J. Huang, C. A. K. Lovell (eds.), UK: Edward Elgar Publishing Limited, 65-89.

[6] Ahn, S, Good, D., and R. C. Sickles (2000), "Estimation of long-run inefficiency levels: a dynamic frontier approach, Econometric Reviews, 19, 461-492.

[7] Aigner, D.J., C.A.K. Lovell, and P. Schmidt (1977), "Formulation and estimation of stochastic frontier models", Journal of Econometrics, 6, 21-3.

[8] Alam, M. Semenick, and R. C. Sickles (2000), "Time series analysis of deregulatory dynamics and technical efficiency: the case of the U. S. airline industry," International Economic Review, 41, 203-218.

[9] Atkinson, S., and C. Cornwell (1994), "Parametric estimation of technical and allocative inefficiency with panel data," International Economic Review, 35, 231-244.

[10] Atkinson, S., and R. Halvorsen (1980), "A test of relative and absolute price efficiency in regulated industries," Review of Economics and Statistics, 62, 81-88.

[11] Baltagi, B. (1995). The Econometric Analysis of Panel Data. New York: John Wiley and Sons.

[12] Banker, R. D., A. Charnes, and W. W. Cooper (1984), "Some models for estimating technical and scaled inefficiencies in data envelopment analysis," Management Science, 32, 16131627 .

[13] Battese G.E., and T. J. Coelli (1988), "Prediction of firm- level technical efficiencies with a generalized frontier production function and panel data", Journal of Econometrics, 38 387-399. 
[14] Battese, G.E. and T.J. Coelli (1992), "Frontier production functions, technical efficiency and panel data: with application to paddy farmers in India", Journal of Productivity Analysis, 3, 153-169.

[15] Battese, G. E., and G. S. Cora (1977), "Estimation of a production frontier model: with application to the pastoral zone of eastern Australia," Australian Journal of Agricultural Economics, 21, 169-179.

[16] Begun, J.M., Hall, W.J., Huang, W.M., and Wellner, J.A. (1983), Information and asymptotic efficiency in parametric-nonparametric models. Annals of Statistics, 11, 432-452.

[17] Berger, A.N., and Humphrey, D.B. (1997), "Efficiency of financial institutions: international survey and directions for future research", European Journal of Operational Research, 98, $175-212$.

[18] Berger, A.N., Kashyap, A.K., and Scalise J.M. (1995), "The transformation of the US banking industry: what a long strange trip it's been", Brookings Papers on Economic Activity, 2, 55-218.

[19] Bickel, P.J., Klaassen, C.A.J., Ritov, Y., and Wellner, J.A. (1993), Efficient and Adaptive Estimation in Non- and Semi-parametric Models. Johns Hopkins University Press, Baltimore.

[20] Charnes, A., Cooper, W. W., and E. L. Rhodes (1978), "Measuring the efficiency of decision making units, European Journal of Operational Research, 2, 429-444.

[21] Coelli, T., Rao, D. S. P., and G. E. Battese (1998), An Introduction to Efficiency and Productivity Analysis (Kluwer Academic Publishers, Boston).

[22] Cornwell, C., Schmidt, P., and Sickles, R.C. (1990), "Production frontiers with crosssectional and time-series variation in efficiency levels,". Journal of Econometrics, 46, 185200.

[23] Debreu, G. (1951), "The coefficient of resource utilization," Econometrica, 19, 273-292.

[24] Färe, R., Grosskopf, S., and C. A. K. Lovell (1985), The Measurement of the Efficiency of Production (Kluwer Academic Publishers, Boston).

[25] Färe, R., Grosskopf, S., and C. A. K. Lovell (1994), Production Frontiers (Cambridge University Press, Cambridge).

[26] Farrell, M. (1957), "The measurement of productive efficiency," Journal of the Royal Statistical Society, Series A, 120, 253-82.

[27] Fernández, C., G. Koop, and M. Steel (2004), "Alternative Efficiency Measures for MultipleOutput Production," mimeo. 
[28] Good, D. H., Nadiri, M. I., and Sickles, R. C. (1991), "The structure of production and technical change in a multiproduct firm: an application to U. S. airlines," National Bureau of Economic Research Working Paper, No. 3939, Cambridge.

[29] Good, D. H., Nadiri, M. I., and Sickles, R. C. (1997), "Index number and factor demand approaches to the estimation of productivity," in Pesaran, M. H., Schmidt, P. (Eds.), Handbook of Applied Economics, Microeconomics, Basil Blackwell, Oxford, 2, 14-80. Reprinted as (1996) National Bureau of Economic Research Working Paper, No. 5790, Cambridge.

[30] Gong, B-H, and R. C. Sickles (1990), "Finite sample properties of stochastic frontier models using panel data," Journal of Productivity Analysis, 229-261.

[31] Gong, B-H, and R. C. Sickles (1992), "A comparison between stochastic frontier and data envelopment methods using panel data," Journal of Econometrics 51, 1992, 259-284.

[32] Greene, W. (1980) "Maximum likelihood estimation of econometric frontier functions," Journal of Econometrics, 13, 27-56.

[33] Greene, W. H. (1997) "Frontier production functions," Chapter 2 of the Handbook of Applied Economics, Volume II-Microeconometrics, edited by M. H. Pesaran and P. Schmidt, Oxford: Basil Blackwell, 1997, 81-166.

[34] Greene, W. H. (2003) "Simulated maximum likelihood estimation of normal-gamma stochastic frontier models," Journal of Productivity Analysis, 19, 179-190.

[35] Hausman, J.A. and Taylor, W.E. (1981), Panel data and unobservable individual effects. Econometrica, 49, 1377-1398.

[36] Ibragimov, I. A., Has'minskii, R. Z. (1981), "Statistical Estimation: Asymptotic Theory," Springer, New York.

[37] Jondrow, J., Lovell, C.A.K., Materov, I.S., and Schmidt, P. (1982), On the estimation of technical inefficiency in stochastic frontier production model. Journal of Econometrics, 19, $233-238$.

[38] Kneip, A.(1994), "Nonparametric estimation of common regressors for similar curve data", Annals of Statistics, 22, 1386-1427.

[39] Kneip, A., Sickles, R. C., and W.H. Song (2003), "On estimating a mixed effects model with applications to the U. S. banking industry, Rice University, mimeo.

[40] Kumbhakar, S. (1987), "The specification of technical and allocative inefficiency in stochastic frontier production and profit functions," Journal of Econometrics, 34, 335-348.

[41] Kumbhakar, S.(1990), "Production frontiers and panel data, and time varying technical inefficiency," Journal of Econometrics, 46, 201-211. 
[42] Lau, L., and P. Yotopoulos (1971), "A test for relative efficiency and application to Indian agriculture," American Economic Review, 61, 94-109.

[43] Lee, Y, and P. Schmidt (1993), "A production frontier model; with flexible temporal variation in technical efficiency," in The measurement of productive efficiency, Fried, H., K. Lovell and S. Schmidt, eds. (Oxford University Press, New York).

[44] Lovell, C. A. K., and S. Kumbakar (2000) Stochastic frontier analysis, New York: Cambridge University Press.

[45] Lovell, C. A. K., and R. C. Sickles (1983), "Testing efficiency hypotheses in joint production: a parametric approach," Review of Economics and Statistics, 65, 51-58.

[46] Meeusen, W. and van den Broeck, J. (1977), "Efficiency estimation from Cobb-Douglas production functions with composed error", International Economic Review, 18, 435-444.

[47] Newey, W.K. (1990), Semiparametric efficiency bounds. Journal of Applied Econometrics, $5,99-136$.

[48] Pagan, P., and Ullah, A. (1999) Non-parametric Econometrics, New York: Cambridge University Press.

[49] Park, B.U. and Simar, L. (1994), Efficient semiparametric estimation in stochastic frontier models. Journal of the American Statistical Association, 89(427), 929-936.

[50] Park, B.U., Sickles, R.C., and Simar, L. (1998), "Stochastic frontiers: a semiparametric approach", Journal of Econometrics, 84, 273-301.

[51] Park, B.U., Sickles, R.C. and Simar, L (2003a), "Semiparametric efficient estimation of ar(1) panel data models," Journal of Econometrics, 117, 279-309.

[52] Park, B.U., Sickles, R.C. and Simar, L (2003b), "Semiparametric efficient estimation of dynamic panel data models," mimeo, Rice University.

[53] Pitt, M. M., and L-F Lee (1981), "The measurement and sources of technical inefficiency in Indonesian weaving industry," Journal of Development Economics, 9, 43-64.

[54] Ritter, C., and L. Simar (1997), "Pitfalls of normal-gamma stochastic frontier models," Journal of Productivity Analysis, 8, 167-182.

[55] Schmidt, P. and Sickles, R.C. (1984), Production frontiers and panel data. Journal of Business and Economic Statistics, 2, 367-374.

[56] Shephard, R. W. (1953), Cost and Production Functions (Princeton University Press, Princeton).

[57] Shephard, R. W. (1970), Theory of Cost and Production Functions, Princeton: Princeton University Press. 
[58] Sickles, R. C ., Good, D. H., and R. Johnson (1986), "Allocative distortions and the regulatory transition of the airline industry," Journal of Econometrics, 33, 143-163.

[59] Simar, L., and P. Wilson (1999), "Estimating and bootstrapping Malmquist indices," European Journal Of Operational Research, 115, 459-471.

[60] Simar, L., and P. Wilson (2000), "A general methodology for bootstrapping in nonparametric frontier models," Journal of applied statistics, 27, 779-802.

[61] Toda, Y. (1976), "Estimation of a cost function when the cost is not minimum: the case of Soviet manufacturing industries: 1958-1971," Review of Economics and Statistics, 58, 259-268. 
[62] van den Broeck, J., G. Koop and J. Osiewalski (1994), Stochastic frontier models: A Bayesian perspective, Journal of Econometrics, 61, 273-303. 


\section{Tables and Figures}

\begin{tabular}{|c|c|c|c|c|c|c|c|c|c|c|c|}
\hline$\overline{\bar{N}}$ & 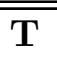 & Within & "GLS & $\overline{\bar{H}-\mathbf{T}}$ & "PSS1 & PSS2W & PSS2G & $\overline{\mathrm{C} \text { CSSW }}$ & C CSSG & 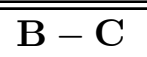 & " KSS \\
\hline 20 & 10 & 0.01076 & 0.05963 & 0.00607 & 0.00606 & 0.00632 & 0.00631 & 0.01816 & 0.00611 & 0.00629 & 0.0137 \\
\hline 20 & 30 & 0.00328 & 0.00239 & 0.00241 & 0.00248 & 0.00268 & 0.00267 & 0.00380 & 0.00208 & 0.00247 & 0.0040 \\
\hline 20 & 50 & 0.00181 & 0.00155 & 0.00156 & 0.00159 & 0.00169 & 0.00169 & 0.00186 & 0.00102 & 0.00156 & 0.0020 \\
\hline 50 & 10 & 0.00395 & 0.00210 & 0.00212 & 0.00231 & 0.00225 & 0.00226 & 0.00635 & 0.00196 & 0.00215 & 0.0047 \\
\hline 50 & 30 & 0.00135 & 0.00106 & 0.00106 & 0.00111 & 0.00111 & 0.00112 & 0.00146 & 0.00088 & 0.00105 & 0.0015 \\
\hline 50 & 50 & 0.00075 & 0.00064 & 0.00064 & 0.00065 & 0.00069 & 0.00069 & 0.00078 & 0.00041 & 0.00064 & 0.000 \\
\hline
\end{tabular}

Table 1: Monte Carlo MSE of the estimators of $\beta$ with no problems

\begin{tabular}{|c|c|c|c|c|c|c|c|c|c|c|c|c|}
\hline$\overline{\mathrm{N}}$ & $\bar{T} \mathbf{T}$ & "Within & GLS & 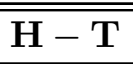 & PSS1 & $\overline{\text { PSS2W }}$ & PSS2G & CSSW & $\overline{\mathrm{CSSG}}$ & $\overline{\mathrm{B}-\mathrm{C}}$ & KSS & $\overline{\overline{\mathrm{D}}}$ \\
\hline 20 & 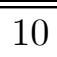 & 0.1381 & "0.1113 & "0.1123 & (20.1118 & (20.1154 & "0.1152 & (0.2794 & (20.2390 & 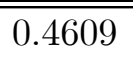 & 0.5872 & 0 . \\
\hline 20 & 30 & 0.0627 & 0.0466 & 0.0474 & 0.0494 & 0.0517 & 0.0517 & 0.1275 & 0.1105 & 0.1359 & 0.2788 & 0. \\
\hline 20 & 50 & 0.0321 & 0.0303 & 0.0303 & 0.0306 & 0.0312 & 0.0312 & 0.0654 & 0.0602 & 0.0832 & 0.2759 & 0 . \\
\hline 50 & 10 & 0.1346 & 0.1200 & 0.1202 & 0.1202 & 0.1227 & 0.1230 & 0.2720 & 0.2548 & 0.4313 & 0.6088 & 0 . \\
\hline 50 & 30 & 0.0525 & 0.0500 & 0.0501 & 0.0501 & 0.0513 & 0.0513 & 0.1161 & 0.1098 & 0.3367 & 0.4157 & 0. \\
\hline 50 & 50 & 0.0416 & 0.0363 & 0.0365 & 0.0373 & 0.0396 & 0.0396 & 0.0978 & 0.0953 & 0.2646 & 0.3739 & 0.7 \\
\hline
\end{tabular}

Table 2: Monte Carlo MSE of the estimators of technical efficiencies with no problems 


\begin{tabular}{|c|c|c|c|c|c|c|c|c|c|c|c|}
\hline N & 1 & & $\overline{\mathrm{LS}}$ & $\begin{array}{l}-T \\
-T\end{array}$ & & & & & & 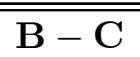 & \\
\hline 20 & $\overline{10}$ & & & & & & & & & & \\
\hline 20 & 30 & & & & & & & & & & \\
\hline 20 & 50 & & & & & & & & & & \\
\hline 50 & 10 & & & & & & & & & & 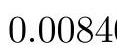 \\
\hline 50 & مי & & & & & & & & & & \\
\hline & 5 & 0334 & 00253 & 00257 & .00270 & .00062 & 0.00062 & 0.0 & .00123 & 0.00253 & .00 \\
\hline
\end{tabular}

Table 3: Monte Carlo MSE of the estimators of $\beta$ with serially correlated disturbances $(\rho=0.8)$.

\begin{tabular}{|c|c|c|c|c|c|c|c|c|c|c|c|c|}
\hline $\mathbf{N}$ & $\overline{T T}$ & Within & 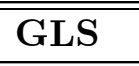 & 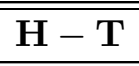 & PSS1 & PSS2W & PSS2G & CSSW & CSSG & $\bar{B}-\mathbf{C}$ & $\overline{\text { KSS }}$ & \\
\hline 20 & 10 & 0 & 611 & 14 & 30 & - & 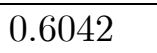 & 71 & 7 & 17 & 4 & \\
\hline 20 & 30 & & & & & & & & & & & \\
\hline 20 & 50 & & & & & & & & & & & \\
\hline 50 & 10 & & & & & & & & & & & \\
\hline 50 & 30 & & & & & & & & & 0.6082 & & \\
\hline 50 & 50 & 0.3653 & 0.3551 & 0.3554 & 0.3567 & 0.3449 & 0.3456 & 0.5760 & 0.5744 & 0.5174 & 0.9146 & \\
\hline
\end{tabular}

Table 4: Monte Carlo MSE of the estimators of technical efficiencies with serially correlated disturbances $(\rho=0.8)$. 


\begin{tabular}{||llllllllllll}
\hline \hline N & T & Within & GLS & H - T & PSS1 & PSS2W & PSS2G & CSSW & CSSG & B - C & KSS \\
\hline \hline 20 & 10 & 0.01076 & 0.00624 & 0.01076 & 0.01116 & 0.00652 & 0.00651 & 0.01816 & 0.00610 & 0.00670 & 0.0137 \\
20 & 30 & 0.00328 & 0.00224 & 0.00329 & 0.00328 & 0.00253 & 0.00253 & 0.00380 & 0.00208 & 0.00230 & 0.0040 \\
20 & 50 & 0.00181 & 0.00155 & 0.00181 & 0.00181 & 0.00169 & 0.00169 & 0.00186 & 0.00102 & 0.00156 & 0.0020 \\
50 & 10 & 0.00395 & 0.00207 & 0.00395 & 0.00405 & 0.00218 & 0.00219 & 0.00640 & 0.00193 & 0.00214 & 0.0047 \\
50 & 30 & 0.00135 & 0.00127 & 0.00135 & 0.00137 & 0.00125 & 0.00125 & 0.00146 & 0.00088 & 0.00126 & 0.0015 \\
50 & 50 & 0.00075 & 0.00057 & 0.00075 & 0.00075 & 0.00061 & 0.00061 & 0.00078 & 0.00041 & 0.00057 & 0.0008 \\
\hline
\end{tabular}

Table 5: Monte Carlo MSE of the estimators of $\beta$ when regressors are correlated with technical efficiency.

\begin{tabular}{|c|c|c|c|c|c|c|c|c|c|c|c|c|}
\hline $\mathbf{N}$ & $\mathbf{T}$ & Within & GLS & $\bar{H}-\mathbf{T}$ & PSS1 & PSS2W & PSS2G & CSSW & CSSG & $\bar{B}-\mathrm{C}$ & KSS & $\mathrm{D}$ \\
\hline 20 & 10 & 0.1447 & 0.1204 & "0.1447 & 0.1458 & 0.1241 & "0.1237 & 0.2835 & 0.2399 & $\overline{0.4431}$ & "0.5973 & \\
\hline 20 & 30 & 0.0618 & 0.0464 & 0.0618 & 0.0619 & 0.0500 & 0.0499 & 0.1267 & 0.1105 & 0.1388 & 0.2768 & 0 . \\
\hline 20 & 50 & 0.0321 & 0.0303 & 0.0321 & 0.0321 & 0.0312 & 0.0312 & 0.0654 & 0.0602 & 0.0832 & 0.2759 & \\
\hline 50 & 10 & 0.1360 & 0.1153 & 0.1360 & 0.1364 & 0.1184 & 0.1184 & 0.2733 & 0.2545 & 0.3684 & 0.6083 & 0 . \\
\hline 50 & 30 & 0.0517 & 0.0550 & 0.0517 & 0.0516 & 0.0543 & 0.0544 & 0.1154 & 0.1100 & 0.3843 & 0.4150 & 0 . \\
\hline 50 & 50 & 0.0434 & 0.0385 & 0.0434 & 0.0433 & 0.0398 & 0.0398 & 0.0987 & 0.0958 & 0.2259 & 0.3754 & 0 \\
\hline
\end{tabular}

Table 6: Monte Carlo MSE of the estimators of technical efficiencies when regressors are correlated with technical efficiency. 


\begin{tabular}{|c|c|c|c|c|c|c|c|c|c|c|c|}
\hline $\mathbf{N}$ & 1 & & $\overline{\mathrm{LS}}$ & 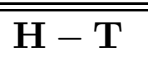 & 51 & & & & & 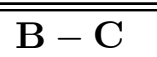 & KSS \\
\hline 20 & $\overline{10}$ & & & & & & & & & & \\
\hline 20 & 30 & & & & & & & & & & \\
\hline 20 & 50 & & & & & & & & & & \\
\hline 50 & 10 & & & & & & & & & & O \\
\hline 50 & 30 & & & & & & & & & & \\
\hline & 50 & 0414 & 0036 & 00362 & .00290 & .00176 & 0.00177 & 0.0 & .00150 & 0.00744 & .00 \\
\hline
\end{tabular}

Table 7: Monte Carlo MSE of the estimators of $\beta$ when technical efficiencies are $\operatorname{ARMA}(1,1)$

\begin{tabular}{|c|c|c|c|c|c|c|c|c|c|c|c|}
\hline $\mathbf{N}$ & $\mathbf{T}$ & Within & GLS & $\mathbf{H}-\mathbf{T}$ & PSS1 & PSS2W & PSS2G & CSSW & CSSG & $\mathrm{B}-\mathrm{C}$ & KSS \\
\hline 20 & 10 & 0.2971 & 0.2721 & 0.2726 & 0.2768 & 0.2717 & 0.2744 & 0.3418 & 0.3088 & 0.4211 & 0.2321 \\
\hline 20 & 30 & 0.4541 & 0.4355 & 0.4369 & 0.4397 & 0.4395 & 0.4390 & 0.4006 & 0.3939 & 0.4504 & 0.1148 \\
\hline 20 & 50 & 0.3036 & 0.2961 & 0.2967 & 0.2973 & 0.2992 & 0.2992 & 0.3183 & 0.3106 & 0.3893 & 0.0842 \\
\hline 50 & 10 & 0.4481 & 0.3941 & 0.3952 & 0.4033 & 0.4095 & 0.4087 & 0.3997 & 0.3572 & 0.4540 & 0.1776 \\
\hline 50 & 30 & 0.5474 & 0.5571 & 0.5566 & 0.5496 & 0.5539 & 0.5537 & 0.5698 & 0.5926 & 0.6081 & 0.0556 \\
\hline 50 & 50 & 0.5719 & 0.5690 & 0.5690 & 0.5687 & 0.5653 & 0.5654 & 0.6515 & 0.6614 & 0.7701 & 0.0455 \\
\hline
\end{tabular}

Table 8: Monte Carlo MSE of ARMA(1,1) time varying technical efficiencies. 


\begin{tabular}{|c|c|c|c|c|c|c|c|c|c|c|c|}
\hline 18 & $\bar{T}$ & $\mathrm{n}$ & $\overline{\mathrm{LS}}$ & $\overline{H-T}$ & S1 & $\bar{N}$ & SS2G & 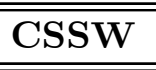 & $\overline{\mathrm{CSSG}}$ & $\overline{\mathrm{B}-\mathrm{C}}$ & $\overline{\mathrm{KSS}}$ \\
\hline 20 & 10 & & & & & & & & & & \\
\hline 2 & 30 & & & & & & & & & & \\
\hline & 50 & & & & & & & & & & \\
\hline & 10 & & & & & & & & & & \\
\hline 50 & 30 & & & & & & & & & & \\
\hline 50 & 50 & 59370 & 0.59370 & 0.59370 & 0.59157 & 0.17622 & 0.13259 & 0.40662 & 0.37150 & 0.55332 & .00 \\
\hline
\end{tabular}

Table 9: Monte Carlo MSE of $\beta$ when the disturbance is serially correlated $(\rho=0.8)$, regressors are correlated with technical efficiency, and technical efficiency follows an $\operatorname{ARMA}(1,1)$ process.

\begin{tabular}{|c|c|c|c|c|c|c|c|c|c|c|c|c|}
\hline $\mathbf{N}$ & $\mathbf{T}$ & Within & GLS & $\mathbf{H}-\mathbf{T}$ & PSS1 & PSS2W & PSS2G & CSSW & CSSG & $\mathrm{B}-\mathrm{C}$ & KSS & $\mathrm{D}$ \\
\hline 20 & 10 & 1.0675 & 0.9277 & 1.0675 & 1.0619 & 1.0923 & 1.1118 & 0.9910 & 0.7810 & 0.7388 & 0.4165 & 1. \\
\hline 20 & 30 & 0.7960 & 0.7393 & 0.7960 & 0.7964 & 0.7924 & 0.7911 & 0.8099 & 0.7737 & 0.7506 & 0.4424 & \\
\hline 20 & 50 & 0.6295 & 0.5606 & 0.6295 & 0.6288 & 0.5679 & 0.5640 & 0.7041 & 0.6734 & 0.6776 & 0.3238 & \\
\hline 50 & 10 & 1.1174 & 1.0430 & 1.1174 & 1.1140 & 1.1109 & 1.1118 & 1.0073 & 0.9338 & 0.9508 & 0.7536 & 0 . \\
\hline 50 & 30 & 0.8550 & 0.8287 & 0.8550 & 0.8551 & 0.7942 & 0.7890 & 0.8744 & 0.8649 & 0.8216 & 0.2693 & 1 . \\
\hline 50 & 50 & 0.8584 & 0.8440 & 0.8584 & 0.8580 & 0.7679 & 0.7588 & 0.8694 & 0.8625 & 0.8002 & 0.2085 & 1 \\
\hline
\end{tabular}

Table 10: Monte Carlo MSE of the estimators of technical efficiencies when the disturbance is serially correlated $(\rho=0.8)$, regressors are correlated with technical efficiency, and technical efficiency follows an $A R M A(1,1)$ process. 


\begin{tabular}{||ccccccccccccc}
\hline \hline N & T & Within & GLS & H - T & PSS1 & PSS2W & PSS2G & CSSW & CSSG & B - C & KSS & D \\
\hline 20 & 10 & 0.9404 & 0.9390 & 0.9388 & 0.9400 & 0.9529 & 0.9540 & 0.8975 & 0.8878 & 0.9328 & 0.1647 & 0. \\
20 & 30 & 0.9539 & 0.9478 & 0.9481 & 0.9493 & 0.9481 & 0.9480 & 0.8924 & 0.8933 & 0.9352 & 0.4274 & 0. \\
20 & 50 & 0.9960 & 0.9959 & 0.9960 & 0.9962 & 0.9972 & 0.9972 & 0.9657 & 0.9652 & 0.9871 & 0.9216 & 0. \\
50 & 10 & 0.9601 & 0.9616 & 0.9616 & 0.9616 & 0.9659 & 0.9660 & 0.9160 & 0.9164 & 0.9439 & 0.3040 & 0. \\
50 & 30 & 0.9875 & 0.9892 & 0.9891 & 0.9888 & 0.9919 & 0.9916 & 0.9347 & 0.9338 & 0.9792 & 0.3065 & 0. \\
50 & 50 & 0.9850 & 0.9865 & 0.9865 & 0.9866 & 0.9872 & 0.9872 & 0.9207 & 0.9191 & 0.9624 & 0.5028 & 0. \\
\hline
\end{tabular}

Table 11: Monte Carlo correlations between true and estimated technical efficiencies with serially correlated disturbances $(\rho=0.8)$

\begin{tabular}{|c|c|c|c|c|c|c|c|c|c|c|c|c|}
\hline $\mathbf{N}$ & $\mathbf{T}$ & Within & GLS & $\mathbf{H}-\mathbf{T}$ & PSS1 & PSS2W & PSS2G & CSSW & CSSG & $\mathrm{B}-\mathrm{C}$ & KSS & $\mathrm{D}$ \\
\hline 20 & $\bar{~} 10$ & 0.9085 & ב0.9505 & "0.9430 & "0.9280 & "0.9070 & 0.9145 & "0.8341 & 0.8491 & 0.9059 & "0.3195 & U \\
\hline 20 & 30 & 0.9550 & 0.9430 & 0.9430 & 0.9430 & 0.9730 & 0.9745 & 0.8629 & 0.8589 & 0.9302 & 0.4248 & \\
\hline 20 & 50 & 0.9880 & 0.9835 & 0.9835 & 0.9835 & 0.9865 & 0.9865 & 0.9440 & 0.9417 & 0.9823 & 0.8806 & \\
\hline 50 & 10 & 0.9169 & 0.9241 & 0.9239 & 0.9241 & 0.9434 & 0.9434 & 0.8609 & 0.8595 & 0.9299 & 0.3513 & 0 . \\
\hline 50 & 30 & 0.9749 & 0.9689 & 0.9689 & 0.9720 & 0.9703 & 0.9702 & 0.8961 & 0.8945 & 0.9594 & 0.3294 & 0 . \\
\hline 50 & 50 & 0.9855 & 0.9851 & 0.9856 & 0.9862 & 0.9867 & 0.9868 & 0.9134 & 0.9103 & 0.9832 & 0.4940 & 0 \\
\hline
\end{tabular}

Table 12: Monte Carlo Spearman rank correlations between true and estimated technical efficiencies with serially correlated disturbances $(\rho=0.8)$. 


\begin{tabular}{|c|c|c|c|c|c|c|c|c|c|c|c|c|}
\hline $\mathbf{N}$ & $\mathbf{T}$ & Within & GLS & $\bar{H}-\mathbf{T}$ & PSS1 & PSS2W & PSS2G & CSSW & CSSG & $\bar{B}-\mathrm{C}$ & KSS & $\mathrm{D}$ \\
\hline 20 & 10 & 0.8227 & 0.8230 & 0.8230 & 0.8228 & 0.8234 & 0.8234 & 0.8820 & 0.8810 & 0.8219 & 0.9937 & 0 . \\
\hline 20 & 30 & 0.3083 & 0.3156 & 0.3151 & 0.3139 & 0.3135 & 0.3138 & 0.5060 & 0.5047 & 0.3540 & 0.9864 & \\
\hline 20 & 50 & 0.3721 & 0.3771 & 0.3769 & 0.3764 & 0.3751 & 0.3754 & 0.3908 & 0.3885 & 0.3584 & 0.9868 & 0 \\
\hline 50 & 10 & 0.7204 & 0.7336 & 0.7335 & 0.7325 & 0.7285 & 0.7286 & 0.8784 & 0.8872 & 0.6726 & 0.9899 & 0 \\
\hline 50 & 30 & 0.5102 & 0.5016 & 0.5020 & 0.5066 & 0.5049 & 0.5049 & 0.5679 & 0.5579 & 0.4648 & 0.9976 & 0 . \\
\hline 50 & 50 & 0.5422 & 0.5423 & 0.5423 & 0.5423 & 0.5419 & 0.5419 & 0.5276 & 0.5208 & 0.4427 & 0.9989 & 0 . \\
\hline
\end{tabular}

Table 13: Monte Carlo correlations between true and estimated technical efficiencies when technical efficiencies are ARMA(1,1)

\begin{tabular}{|c|c|c|c|c|c|c|c|c|c|c|c|c|}
\hline 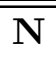 & $\bar{T} \mathbf{T}$ & Within & GLS & $\overline{\mathbf{H}-\mathbf{T}}$ & 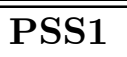 & PSS2W & PSS2G & $\overline{\mathrm{CSSW}}$ & CSSG & $\bar{B}-\mathrm{C}$ & KSS & $\overline{\bar{D}}$ \\
\hline 20 & $\bar{~} 10$ & 0.5308 & 0.5308 & 0.5308 & 0.5309 & ב0.5336 & 0.5336 & "0.7936 & "0.7938 & 0.5539 & 0.9971 & 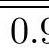 \\
\hline 20 & 30 & 0.2488 & 0.2481 & 0.2488 & 0.2488 & 0.2488 & 0.2488 & 0.4721 & 0.4696 & 0.3761 & 0.9958 & \\
\hline 20 & 50 & 0.3605 & 0.3606 & 0.3606 & 0.3606 & 0.3606 & 0.3606 & 0.3832 & 0.3801 & 0.3695 & 0.9906 & 0. \\
\hline 50 & 10 & 0.6517 & 0.6512 & 0.6512 & 0.6520 & 0.6517 & 0.6517 & 0.8001 & 0.7916 & 0.6587 & 0.9972 & 0 . \\
\hline 50 & 30 & 0.4370 & 0.4358 & 0.4359 & 0.4367 & 0.4365 & 0.4365 & 0.5745 & 0.5709 & 0.4471 & 0.9993 & 0 . \\
\hline 50 & 50 & 0.5055 & 0.5055 & 0.5055 & 0.5055 & 0.5055 & 0.5055 & 0.6641 & 0.6629 & 0.5862 & 0.9998 & 0 . \\
\hline
\end{tabular}

Table 14: Monte Carlo Spearman rank correlations between true and estimated technical efficiencies when technical efficiencies are $\operatorname{ARMA}(1,1)$ 


\begin{tabular}{|c|c|c|c|c|c|c|c|c|c|c|c|c|}
\hline 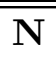 & 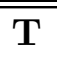 & Within & $\overline{\text { GLS }}$ & $\overline{\bar{H}-\mathbf{T}}$ & PSS1 & PSS2W & PSS2G & C CSSW & C CSSG & 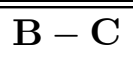 & KSS & $\overline{\overline{\mathrm{D}}}$ \\
\hline 20 & 10 & 0.3254 & 0.4552 & 0.3254 & 0.3260 & 0.3085 & 0.2988 & 0.4347 & 0.6957 & 0.4277 & 0.9262 & 0. \\
\hline 20 & 30 & 0.0619 & 0.1083 & 0.0619 & 0.0618 & 0.1271 & 0.1280 & 0.1570 & 0.2412 & 0.1437 & 0.8697 & \\
\hline 20 & 50 & 0.1837 & 0.2403 & 0.1837 & 0.1842 & 0.2111 & 0.2145 & 0.2016 & 0.2443 & 0.2254 & 0.8436 & 0 . \\
\hline 50 & 10 & 0.0499 & 0.1446 & 0.0499 & 0.0540 & 0.0475 & 0.0465 & 0.2895 & 0.4664 & 0.1250 & 0.6069 & c \\
\hline 50 & 30 & 0.3178 & 0.3426 & 0.3178 & 0.3178 & 0.3678 & 0.3732 & 0.3660 & 0.3806 & 0.3126 & 0.9373 & \\
\hline 50 & 50 & 0.3907 & 0.4010 & 0.3907 & 0.3909 & 0.4597 & 0.4698 & 0.4304 & 0.4368 & 0.3900 & 0.9891 & U. \\
\hline
\end{tabular}

Table 15: Monte Carlo correlations between true and estimated technical efficiencies when disturbance is serially correlated $(\rho=0.8)$, regressors are correlated with technical efficiency, and technical efficiencies are $\operatorname{ARMA}(1,1)$

\begin{tabular}{|c|c|c|c|c|c|c|c|c|c|c|c|c|}
\hline $\mathbf{N}$ & $\mathbf{T}$ & Within & GLS & $\mathbf{H}-\mathbf{T}$ & PSS1 & PSS2W & PSS2G & CSSW & CSSG & $\mathrm{B}-\mathrm{C}$ & KSS & $\mathrm{D}$ \\
\hline 20 & 10 & 0.2909 & 0.3546 & 0.2909 & 0.2955 & 0.3384 & 0.3439 & 0.4367 & 0.5855 & 0.3576 & 0.9140 & 0 . \\
\hline 20 & 30 & 0.1124 & 0.1433 & 0.1124 & 0.1124 & 0.1528 & 0.1531 & 0.2465 & 0.3240 & 0.2044 & 0.8865 & 0 \\
\hline 20 & 50 & 0.1710 & 0.2197 & 0.1710 & 0.1710 & 0.1857 & 0.1860 & 0.1871 & 0.2336 & 0.2046 & 0.8458 & 0.7 \\
\hline 50 & 10 & 0.3058 & 0.3834 & 0.3058 & 0.3130 & 0.3048 & 0.3050 & 0.5325 & 0.6389 & 0.3649 & 0.8370 & 0.8 \\
\hline 50 & 30 & 0.2363 & 0.2508 & 0.2363 & 0.2363 & 0.2753 & 0.2841 & 0.3567 & 0.3830 & 0.2398 & 0.9715 & 0 . \\
\hline 50 & 50 & 0.2299 & 0.2348 & 0.2299 & 0.2299 & 0.3784 & 0.4001 & 0.4229 & 0.4433 & 0.2469 & 0.9957 & 0.7 \\
\hline
\end{tabular}

Table 16: Monte Carlo Spearman rank correlations between true and estimated technical efficiencies when disturbance is serially correlated $(\rho=0.8)$, regressors are correlated with technical efficiency, and technical efficiencies are $A R M A(1,1)$ 


\begin{tabular}{|c|c|c|c|c|c|c|c|c|c|c|c|}
\hline True & Within & GLS & $\mathbf{H}-\mathbf{T}$ & PSS1 & PSS2W & PSS2G & CSSW & CSSG & B $-C$ & KSS & $\mathrm{DE}$ \\
\hline & (20.1837 & (0.2403 & 0.1837 & "0.1842 & (0.2111 & "0.2145 & (0.2016 & "0.2443 & (0.2254 & "0.8436 & \\
\hline & . & 0.9790 & 1.0000 & 1.0000 & 0.9932 & 0.9911 & 0.9568 & 0.9292 & 0.9808 & 0.2237 & \\
\hline & & . & 0.9790 & 0.9794 & 0.9942 & 0.9952 & 0.9347 & 0.9350 & 0.9934 & 0.2987 & \\
\hline & & & $\cdot$ & 1.000 & 0.9932 & 0.9911 & 0.9568 & 0.9292 & 0.9808 & 0.2257 & \\
\hline & & & & . & 0.9935 & 0.9914 & 0.95568 & 0.9295 & 0.9811 & 0.2264 & \\
\hline & & & & & . & 0.9996 & 0.9510 & 0.9378 & 0.9929 & 0.2607 & \\
\hline & & & & & & . & 0.9495 & 0.9384 & 0.9937 & 0.2657 & 0 . \\
\hline & & & & & & & $\cdot$ & 0.9874 & 0.9452 & 0.2362 & 0.1 \\
\hline & & & & & & & & $\cdot$ & 0.9413 & 0.2920 & \\
\hline & & & & & & & & & & 0.2815 & \\
\hline & & & & & & & & & & & \\
\hline
\end{tabular}

Table 17: Monte Carlo correlations of technical efficiencies when the disturbance is serially correlated $(\rho=0.8)$, regressors are correlated with technical efficiency, and technical efficiencies are $\operatorname{ARMA}(1,1)(N=20, T=50)$. 


\begin{tabular}{|c|c|c|c|c|c|c|c|c|c|c|c|}
\hline True & Within & GLS & $\mathbf{H}-\mathbf{T}$ & PSS1 & PSS2W & PSS2G & CSSW & CSSG & B $-C$ & KSS & $\mathrm{DE}$ \\
\hline & "0.1710 & (0.2197 & 0.1710 & "0.1710 & (0.1857 & "0.1860 & "0.1871 & "0.2336 & (0.2046 & "0.8458 & \\
\hline & . & 0.9805 & 1.0000 & 1.0000 & 0.9880 & 0.9910 & 0.9598 & 0.9271 & 0.9810 & 0.2182 & 0 . \\
\hline & & . & 0.9805 & 0.9805 & 0.9835 & 0.9850 & 0.9390 & 0.9300 & 0.9909 & 0.2793 & \\
\hline & & & $\cdot$ & 1.0000 & 0.9880 & 0.9910 & 0.9598 & 0.9271 & 0.9810 & 0.2182 & \\
\hline & & & & . & 9.9880 & 0.9910 & 0.9598 & 0.9271 & 0.9810 & 0.2182 & \\
\hline & & & & & . & 0.9955 & 0.9544 & 0.9313 & 0.9823 & 0.2372 & \\
\hline & & & & & & . & 0.9560 & 0.9336 & 0.9847 & 0.2380 & \\
\hline & & & & & & & 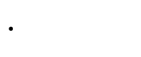 & 0.9847 & 0.9524 & 0.2279 & \\
\hline & & & & & & & & . & 0.9421 & 0.2815 & \\
\hline & & & & & & & & & & 0.2685 & 0 . \\
\hline & & & & & & & & & & & 0.73 \\
\hline
\end{tabular}

Table 18: Monte Carlo Spearman rank correlations of technical efficiencies when the disturbance is serially correlated $(\rho=0.8)$, regressors are correlated with technical efficiency, and technical efficiencies are $\operatorname{ARMA}(1,1)(N=20, T=50)$. 